Research Article

\title{
Intestinal Carriage of Extended-Spectrum $\beta$-Lactamase- (ESBL-) Possessing Escherichia coli and Klebsiella Species among Nepalese Health Science and Non-Health Science Students
}

\author{
Bhawana Sapkota, ${ }^{1}$ Santosh K. Yadav $\mathbb{D}^{2},{ }^{2}$ Gunaraj Dhungana, ${ }^{1}$ Shamshul Ansari, ${ }^{3}$ \\ and Shyam K. Mishra $\mathbb{D}^{4,5}$ \\ ${ }^{1}$ Department of Medical Laboratory Technology, JF Institute of Health Sciences, Kathmandu, Nepal \\ ${ }^{2}$ Department of Microbiology, Rajarshi Janak University, Janakpurdham, Nepal \\ ${ }^{3}$ Department of Microbiology, Chitwan Medical College, School of Medicine, Bharatpur, Nepal \\ ${ }^{4}$ Department of Microbiology, Maharajgunj Medical Campus, Institute of Medicine, Tribhuvan University, Kathmandu, Nepal \\ ${ }^{5}$ School of Optometry and Vision Science, University of New South Wales Sydney, Kensington, Australia
}

Correspondence should be addressed to Shyam K. Mishra; smishra7@hotmail.com

Received 21 May 2020; Revised 3 November 2020; Accepted 27 March 2021; Published 9 April 2021

Academic Editor: Maria De Francesco

Copyright (c) 2021 Bhawana Sapkota et al. This is an open access article distributed under the Creative Commons Attribution License, which permits unrestricted use, distribution, and reproduction in any medium, provided the original work is properly cited.

\begin{abstract}
Infections due to extended-spectrum $\beta$-lactamase- (ESBL-) producing Gram-negative bacteria have led to increased mortality, morbidity, and economic burden worldwide. These bacteria can colonize the healthy intestine of human beings and can disseminate in communities and hospital. This study aimed to investigate the prevalence of fecal carriage of ESBL-producing Escherichia coli and Klebsiella species among health science (HS) and non-health science (NHS) students. This descriptive crosssectional study was conducted on 104 HS and 104 NHS students in which one stool sample from each student was collected and processed for bacterial culture and sensitivity testing according to standard bacteriological procedures. Each morphotype was identified and characterized phenotypically. The antimicrobial sensitivity profile of bacterial isolates was determined by the Kirby-Bauer disk diffusion technique. ESBL production was tested by combination disk method as recommended by the Clinical and Laboratory Standards Institute. Out of 208 stool samples, E. coli and Klebsiella spp. were recovered from 203 (86.8\%) and 31 $(13.2 \%)$ stool samples, respectively. Among those 234 isolates, 69 were positive for ESBL which included E. coli $(n=66,95.7 \%)$ and Klebsiella spp. ( $n=3,4.3 \%)$. Fifty (42.4\%) out of 118 isolates from HS students and $19(16.4 \%)$ out of 116 from NHS students were colonized by ESBL-producers. Compared to non-ESBL producers, a higher number of ESBL-producing isolates were resistant to ciprofloxacin ( $14.5 \%$ vs. $1.8 \%, p<0.001)$, cotrimoxazole (59.4\% vs. $16.4 \%, p<0.001)$, and amikacin $(10.1 \%$ vs $4.2 \%, p<0.001)$. All E. coli and Klebsiella species isolates were susceptible to meropenem. The prevalence of fecal carriage of ESBL-producing bacteria was higher in HS students; however, there was a considerable number of these strains colonizing NHS students as well. This "iceberg phenomenon" of asymptomatic carriage of ESBL-producing pathogens might act as a source of infection in both the community and hospitals. Therefore, surveillance of carriage of drug-resistant bacteria should be performed regularly.
\end{abstract}

\section{Introduction}

The gut microbiota is an extremely complex community composed of both aerobic and anaerobic bacterial populations. However, in healthy humans, the gut microbiota is relatively stable and ingested microorganisms are cleared easily due to the presence of commensal microbiota [1].
Antibiotic resistance in Escherichia coli (E. coli) and Klebsiella species (common intestinal members of the microbiota) to third-generation cephalosporins are a major threat in hospitalized patients as well as in community populations. This resistance is mediated mainly by acquired extended-spectrum $\beta$-lactamase (ESBL) enzymes, which have the potential to hydrolyze penicillins, cephalosporins, and monobactams [2]. 
Rates of intestinal colonization by ESBL-producing E. coli and Klebsiella species have increased dramatically worldwide. This increased prevalence has been associated with the dissemination of specific clones and plasmids harboring ESBL genes. The spread of ESBL-producing organisms to healthy community residents is one of the most threatening epidemiological problems worldwide [3]. The rapid emergence of ESBL-producing bacteria results in a therapeutic burden that generates economic and public health concerns to healthcare systems globally. Thus, it is necessary to identify high-risk populations to help reduce this problem [4].

Although some data are available on the intestinal carriage of ESBL-producing Enterobacterales in community populations in South Asian countries [5-8], there is a lack of published estimates of intestinal carriage of ESBL-producing E. coli and Klebsiella species among health science (HS) and non-health science (NHS) students. Therefore, it was hypothesized that the HS students who are concerned with hospital patients and community populations would harbor greater numbers of antibiotic-resistant ESBL-producing E. coli and Klebsiella species as part of their commensal intestinal microbiota. They may also disseminate these bacterial populations causing hospital-associated as well as community-acquired infections. This study focused on the intestinal (fecal) carriage rate of ESBL-producing E. coli and Klebsiella species among HS and NHS students of a health science institute of central Nepal which provides undergraduate courses in nursing, pharmacy, and medical laboratory technology.

\section{Materials and Methods}

2.1. Study Design and Study Population. This descriptive cross-sectional study was conducted over six months among the HS and NHS students of the JF Institute of Health Sciences, Nepal, and microbiological procedures were carried out at the microbiology department of the JF Institute of Health Sciences/ Little Angels College of Higher Studies (LACHS). From July to December 2017, a total of 208 students (104 each from HS and NHS categories) were enrolled. The HS students who participated in this study were from BNS (Bachelor of Nursing Science), B.Sc. Nursing (Bachelor of Science in Nursing), and B.Sc. MLT (Bachelor of Science in Medical Laboratory Technology) programs. Both BNS and B.Sc. Nursing fall under the nursing program, but they were recognized as different health science student groups in this study because only those people who have completed the certificate-level course in nursing can apply for the BNS, whereas for B.Sc. Nursing, candidates who have completed a higher secondary level in science can apply. All groups of HS students had frequent clinical posting in hospitals before the sampling period. Student volunteers not from the health science program of LACHS and who were interested to participate in the study were selected as the NHS students. The demographics of the participating students including previous antibiotic exposure or infections were recorded.

2.2. Inclusion and Exclusion Criteria. Students without prior antibiotic exposure for at least three months before study enrollment and those without infectious diseases were included in this study. The exclusion criteria included students who were under antibiotic therapy.

2.3. Stool Sample Collection and Processing. During the study, a total of 208 non-duplicate fecal samples were collected comprising of 104 each from the HS and NHS groups. After obtaining the written consent in local and English languages, each participant was provided with a clean, sterile, leak-proof, screw-capped plastic container with a plastic spoon and was instructed in the collection of a fresh stool sample. A single stool sample was collected from each student. Then, the fecal samples were processed for isolation and identification of E. coli and Klebsiella species according to standard microbiological methods recommended by the American Society for Microbiology (ASM) [9]. For this, each stool sample was inoculated onto a MacConkey agar plate and incubated aerobically at $37^{\circ} \mathrm{C}$ for 24 hours.

2.4. Identification of Bacterial Isolates. After incubation, different bacterial morphotypes were selected and further processed for identification by observation of colony characteristics, Gram's staining, motility, and different biochemical tests (catalase test, oxidase test, triple sugar iron agar test, indole production, citrate utilization, and urea hydrolysis) [9]. Those isolates identified as E. coli or Klebsiella species were subjected to antibiotic sensitivity testing and detection of ESBL production.

2.5. Antibiotic Sensitivity Testing. Antibiotic sensitivity testing (AST) was performed by the Kirby-Bauer disk diffusion method, and results were interpreted according to Clinical and Laboratory Standards Institute (CLSI) guidelines [10]. A loop was charged with at least 3-5 well-isolated colonies of bacteria, and these bacteria were transferred to a tube containing $5 \mathrm{ml}$ of peptone water. The peptone water tube was incubated at $37^{\circ} \mathrm{C}$ until its turbidity matched a 0.5 McFarland standard. A sterile cotton swab was dipped into that peptone water, and the swab was rotated several times and then pressed firmly on the inner sidewall of the tube to remove excess inoculum from the swab. Then, the entire surface of a Mueller-Hinton agar (MHA) (HiMedia, India) plate was inoculated with the swab (by rotating the plate by $60^{\circ}$ between streaking) to produce a lawn culture. The plates were left to dry for 10 minutes at room temperature with the lid closed and then antibiotic disks (ampicillin $(10 \mu \mathrm{g})$, piperacillin-tazobactam $(100 / 10 \mu \mathrm{g})$, cefotaxime $(30 \mu \mathrm{g})$, ceftazidime $(30 \mu \mathrm{g})$, meropenem $(10 \mu \mathrm{g})$, ciprofloxacin $(5 \mu \mathrm{g})$, cotrimoxazole $(25 \mu \mathrm{g})$, and amikacin $(30 \mu \mathrm{g}))$ (HiMedia, India) were placed on the surface of the agar plate and incubated in ambient air for $16-18$ hours at $37^{\circ} \mathrm{C}$. After incubation, the diameter of the zone of inhibition (ZOI) around each disk was measured and the isolate was classified as sensitive, of intermediate sensitivity, or resistant to each antibiotic using the CLSI zone size interpretation chart. Escherichia coli ATCC 25922 was used as the quality control strain for the test [10]. 
2.6. Detection of Extended-Spectrum- $\beta$-Lactamase- (ESBL-) Producing Isolates. Extended-spectrum- $\beta$-lactamase production in E. coli and Klebsiella species was detected following CLSI guidelines [10].

Screening Test. For ESBL screening, bacterial isolates were examined for their susceptibility to third-generation cephalosporins using ceftazidime $(30 \mu \mathrm{g})$ and cefotaxime $(30 \mu \mathrm{g})$ disks. If the ZOI was $\leq 22 \mathrm{~mm}$ for ceftazidime and/or $\leq 27 \mathrm{~mm}$ for cefotaxime, the isolate was considered a potential ESBL-producer [10].

Confirmatory Test. Confirmation of ESBL production was carried out using the combination disk test (CDT). In this test, ceftazidime $(30 \mu \mathrm{g})$ disk alone and in combination with clavulanic acid $(30 / 10 \mu \mathrm{g})$ or cefotaxime $(30 \mu \mathrm{g})$ disk alone and in combination with clavulanic acid $(30 / 10 \mu \mathrm{g})$ were applied onto a plate of MHA previously inoculated with the test strain. The plate was incubated in ambient air for 16-18 hours at $37^{\circ} \mathrm{C}$. Isolates showing an increase of $\geq 5 \mathrm{~mm}$ in the zone of inhibition (ZOI) for either antimicrobial agent tested in combination with clavulanic acid versus the zone diameter of the agent when tested alone were considered positive for ESBL production. For standardization of the ESBL detection test, K. pneumoniae ATCC 700603 and E. coli ATCC 25922 were used as positive and negative controls, respectively [10].

2.7. Statistical Analysis. Data analysis was carried out using the SPSS version 16.0 and interpreted according to frequency distribution and percentage. Pearson's chi-square test was used to determine correlations between variables. $p$ values $\leq 0.05(95 \% \mathrm{CI})$ were considered to be statistically significant.

2.8. Ethical Statement. Ethical approval was obtained from the Ethical Review Board of Nepal Health Research Council, Kathmandu, Nepal (Reg. No. 254/2017). Informed consent was obtained from each student before enrollment in the study.

\section{Results}

3.1. Distribution of Enrolled Students and Bacterial Isolates. The total number of students who participated in the study was 208 which was equally split between the HS and NHS students (104 in each). Among all the students, 77 were male and 131 were female, giving a male to female ratio of 0.59 . The mean age of the study participants was 21.37 years $(\mathrm{SD} \pm 1.62)$ ranging from 18 years to 26 years.

A total of 234 isolates were grown. Of these, $86.8 \%$ were identified as E. coli $(n=203), 8.5 \%$ as Klebsiella pneumoniae $(n=20)$, and $4.7 \%$ as Klebsiella oxytoca $(n=11)$. One hundred and one $(49.8 \%)$ and $102(50.2 \%)$ isolates of $E$. coli were recovered from $\mathrm{HS}$ and NHS students, respectively. The recovery rate of Klebsiella spp. was $54.8 \%(n=17)$ and $45.2 \%(n=14)$ from HS and NHS students, respectively (Table 1). Among these students, 60 ,
TABle 1: Distribution of E. coli and Klebsiella species among HS and NHS students.

\begin{tabular}{lccc}
\hline \multirow{2}{*}{ Bacterial isolates } & \multicolumn{3}{c}{ Number of isolates (\%) } \\
& HS students & NHS students & Total \\
\hline Escherichia coli & $101(49.8)$ & $102(50.2)$ & 203 \\
Klebsiella pneumoniae & $8(40.0)$ & $12(60.0)$ & 20 \\
Klebsiella oxytoca & $9(81.8)$ & $2(18.2)$ & 11 \\
\hline Total & $118(50.4)$ & $116(49.6)$ & 234 \\
\hline
\end{tabular}

25, and 19 were from B.Sc. Nursing, BNS, and B.Sc. MLT programs, respectively. Out of $101 \mathrm{E}$. coli isolated from HS students, $56.4 \% \quad(n=57), 24.8 \% \quad(n=25)$, and $18.8 \%$ $(n=19)$ were recovered from B.Sc. Nursing, BNS, and B.Sc. MLT students, respectively. Similarly, out of 17 Klebsiella species from HS students, $64.8 \%(n=11)$ was recovered from B.Sc. Nursing and $17.6 \%(n=3)$ from each BNS and B.Sc. MLT students (Table 2).

3.2. ESBL Carriage among Health Science and Non-Health Science Students. The overall prevalence of fecal carriage of ESBL-producing isolates among the students was $29.5 \%$. Most (50 out of $118 ; 42.4 \%$ ) isolates were from HS students and 19 out of $116(16.4 \%)$ isolates were from NHS students. The rate of ESBL production among E. coli and Klebsiella species was $32.5 \%$ and $9.7 \%$, respectively. ESBL production in E. coli was highest among HS students $(46.5 \%, p<0.001)$. Only a few isolates of Klebsiella species from HS students were ESBL-producers $(n=3,17.6 \%)$. Among the NHS students, $18.6 \%(n=19)$ E. coli were ESBL-producers, whereas none of the isolates of Klebsiella species produced ESBL (Table 3). Among the HS students, $45.6 \%, 56.0 \%$, and $36.8 \% \mathrm{E}$. coli were ESBL positive from B.Sc. Nursing, BNS, and B.Sc. MLT students, respectively. Similarly, 33.3\% (one out of three) and $18.2 \%$ (two out of 11) Klebsiella spp. isolates from BNS and B.Sc. Nursing students, respectively, were ESBL-producers (Table 4). None of the students harbored both ESBL-producing E. coli and Klebsiella spp.

3.3. Antibiotic Sensitivity Profiles of Bacterial Isolates. Both E. coli and Klebsiella spp. isolates from HS students had higher resistance rates than those from NHS students. Isolates of $E$. coli from HS students had increased resistance to ampicillin $(71.3 \%$ vs $51.0 \%, p=0.001)$, third-generation cephalosporins $(60.4 \%$ vs $30.4 \%, p<0.001)$, and cotrimoxazole ( $39.6 \%$ vs $30.4 \%, p=0.005)$. Similarly, Klebsiella species from HS students had higher resistance rates to cotrimoxazole $(29.4 \%)$ or to third-generation cephalosporins $(23.5 \%)$ as all of the Klebsiella species isolates from NHS students were sensitive to all antibiotics except cotrimoxazole. All the isolates of E. coli and Klebsiella species from both HS and NHS students were susceptible to meropenem (Table 5). A higher number of ESBL-producers were resistant to ciprofloxacin $(14.5 \%$ vs. $1.8 \%, p<0.001)$, cotrimoxazole (59.4\% vs. $16.4 \%, p<0.001)$, and amikacin $(10.1 \%$ vs $4.2 \%, p<0.001$ ) (Table 6 ). 
Table 2: Distribution of E. coli and Klebsiella species among different programs of HS students.

\begin{tabular}{|c|c|c|c|c|}
\hline \multirow{2}{*}{ Bacterial isolates } & \multicolumn{4}{|c|}{ Number of isolates (\%) } \\
\hline & B.Sc. Nursing & BNS & B.Sc. MLT & Total \\
\hline Escherichia coli & $\begin{array}{c}57 \\
(56.4)\end{array}$ & $\begin{array}{c}25 \\
(24.8)\end{array}$ & $\begin{array}{c}19 \\
(18.8)\end{array}$ & $\begin{array}{c}101 \\
(85.6)\end{array}$ \\
\hline Klebsiella pneumoniae & $3(37.5)$ & $3(37.5)$ & $2(25.0)$ & $8(6.8)$ \\
\hline Klebsiella oxytoca & $8(88.9)$ & $0(0)$ & $1(11.1)$ & $9(7.6)$ \\
\hline Total & $68(57.6)$ & $28(23.7)$ & $22(18.7)$ & $118(100)$ \\
\hline
\end{tabular}

TABLE 3: Prevalence of fecal carriage of ESBL-producing isolates.

\begin{tabular}{|c|c|c|c|c|c|c|}
\hline \multirow{2}{*}{ Bacterial isolates } & \multicolumn{2}{|c|}{ Number of isolates } & \multicolumn{3}{|c|}{ Number of ESBL producers (\%) } & \multirow{2}{*}{$p$ value } \\
\hline & HS students & NHS students & HS students & NHS students & Total & \\
\hline E. $\operatorname{coli}(n=203)$ & 101 & 102 & $47(46.5)$ & $19(18.6)$ & $66(32.5)$ & $<0.001$ \\
\hline Klebsiella species $(n=31)$ & 17 & 14 & $3(17.6)$ & $0(0)$ & $3(9.7)$ & 0.10 \\
\hline Total $(N=234)$ & 118 & 116 & $50(42.4)$ & $19(16.4)$ & $69(29.5)$ & $<0.001$ \\
\hline
\end{tabular}

TABLE 4: ESBL carriage among different programs of HS students.

\begin{tabular}{|c|c|c|c|c|}
\hline \multirow{2}{*}{ Bacterial isolates } & \multicolumn{4}{|c|}{ Number of ESBL producers out of total number of isolates (\%) } \\
\hline & B.Sc. Nursing & BNS & B.Sc. MLT & Total \\
\hline E. coli & $26 / 57(45.6)$ & $14 / 25(56.0)$ & $7 / 19(36.8)$ & $47 / 101(46.5)$ \\
\hline Klebsiella species & $2 / 11(18.2)$ & $1 / 3(33.3)$ & $0 / 3(0)$ & $3 / 17(12.5)$ \\
\hline Total $(n=118)$ & $28 / 68(41.2)$ & $15 / 28(22.1)$ & $7 / 22(31.8)$ & $50 / 118(42.4)$ \\
\hline
\end{tabular}

TABLE 5: Antibiotic resistance rates of bacterial isolates among HS and NHS students.

\begin{tabular}{lcccc}
\hline \multirow{2}{*}{ Antibiotics } & \multicolumn{2}{c}{ Escherichia coli } & \multicolumn{2}{c}{ Klebsiella species } \\
& HS students $(n=101)$ & NHS students $(n=102)$ & HS students $(n=17)$ & NHS students $(n=14)$ \\
\hline Ampicillin & $72(71.3 \%)$ & $52(51.0 \%)$ & NA & NA \\
Piperacillin-tazobactam & $2(2.0 \%)$ & $1(1.0 \%)$ & $1(5.9 \%)$ & $0(0)$ \\
Cefotaxime & $61(60.4 \%)$ & $31(30.4 \%)$ & $4(23.5 \%)$ & $0(0)$ \\
Ceftazidime & $61(60.4 \%)$ & $31(30.4 \%)$ & $4(23.5 \%)$ & $0(0)$ \\
Ciprofloxacin & $5(5.0 \%)$ & $7(6.9 \%)$ & $1(5.9 \%)$ & $0(0)$ \\
Cotrimoxazole & $40(39.6 \%)$ & $22(21.6 \%)$ & $5(29.4 \%)$ & $1(7.1 \%)$ \\
Amikacin & $4(4.0 \%)$ & $9(8.8 \%)$ & $1(5.9 \%)$ & $0(0)$ \\
Meropenem & $0(0)$ & $0(0)$ & $0(0)$ & $0(0)$ \\
\hline
\end{tabular}

NA, not applicable.

TABLE 6: Antimicrobial resistance rates of ESBL-producing and non-producing isolates.

\begin{tabular}{lccc}
\hline Antibiotics & ESBL-non-producers $(n=165)$ & ESBL producers $(n=69)$ & $p$ value \\
\hline Piperacillin-tazobactam & $2(1.2 \%)$ & $2(2.9 \%)$ & 0.08 \\
Ciprofloxacin & $3(1.8 \%)$ & $10(14.5 \%)$ & $<0.001$ \\
Cotrimoxazole & $27(16.4 \%)$ & $41(59.4 \%)$ & $<0.001$ \\
Amikacin & $7(4.2 \%)$ & $7(10.1 \%)$ & $<0.001$ \\
Meropenem & $0(0)$ & $0(0)$ & \\
\hline
\end{tabular}

\section{Discussion}

Antimicrobial resistance is a major public health issue which is likely to worsen in the coming decades. Gram-negative bacteria, particularly E. coli and Klebsiella species, are now often resistant to third-generation cephalosporins, and one of the reasons behind this is their capacity to produce ESBLs [1]. People colonized with ESBL-producing Gram-negative bacteria may transmit the resistant strains to other individuals, including hospitalized patients. High rates of intestinal carriage of such resistant bacteria have been described from developing countries [11, 12]. Various studies conducted worldwide have shown a dramatically elevated prevalence of ESBL-producing E. coli and Klebsiella species in fecal samples of healthy individuals $[3,13]$. This study determined the fecal carriage rate of ESBL-producing E. coli and Klebsiella species among different clusters of students at the JF Institute of Health Sciences/Little Angels College of Higher Students in Nepal. 
In this study, the overall fecal carriage rate of ESBLproducing E. coli and Klebsiella species among the enrolled students was $29.5 \%$ which is similar to previous reports of $25-38 \%$ ESBL carriage rate in healthy populations in Nepal [6] and Chad [14]. However, the result is much higher than some other reports from different parts of the world, for instance, $6.7 \%$ of student volunteers from Cameroon [12], $6.4 \%$ of healthy adults from Japan [15], 2.6\% of medical students in Hungary [16], and 3.5\% of healthy children in the USA [17]. A similar study conducted in Nepal in 2016, which focused on the detection of ESBL-producing Enterobacterales among healthy adult volunteers of a health science college, found a relatively low rate of $9.8 \%$ for fecal carriage of ESBL-producing E. coli and Klebsiella species [5]. A meta-analysis of data on ESBL carriage among healthy individuals found there was an increase in ESBL colonization of 5.38\% per year [18] and this may have contributed to the high level of ESBL carriage found in the current study compared. Another reason for the higher prevalence of ESBL-producing isolates among HS students in the current study could have been due to the frequent visit of these student groups to healthcare settings which resulted in the acquisition of such bacteria from different hospital environments, patients, and healthcare workers.

In the current study, the majority of ESBL-producing Enterobacterales were E. coli (32.5\%) with a higher rate of ESBL-producing E. coli isolated from HS students (46.5\%, 47/101). All the ESBL-producing Klebsiella isolates were recovered from HS students $(17.6 \%, 3 / 17)$. A variable rate of fecal carriage of ESBL-producing E. coli and Klebsiella species has been reported in community settings from different countries. The rate of ESBL production in E. coli was higher from a previous study in Nepal (77.8\%) [6] and from one study from Egypt (62.7\%) [19]. However, lower rates of ESBL carriage also have been reported from Nepal (7.87\%) [5], France (6\%) [20], UK (5.95\%) [4], and Tunisia (7.3\%) [21]. Previous reports have found $14.2 \%$ and $20.5 \%$ ESBL-producing Klebsiella species from the gut of Nepalese healthy population and student volunteers, respectively $[5,6]$. These differences are likely to have been produced by different rates of exposure to antibiotics or to the bacterial isolates in the different populations. The students from the BNS program harbored a greater number of ESBL-producing E. coli (56.0\%) and Klebsiella species (33.3\%) than other groups of HS and NHS students, and this may also be attributed to a more frequent exposure of BNS students to ESBL-producing bacteria in hospitals before their enrollment in the BNS program as they would have undertaken job experience in hospitals prior to their enrollment in BNS program.

The current study also focused on the sensitivity of all E. coli and Klebsiella species isolates to a set of antimicrobial agents. When compared with non-ESBL producers, the ESBL-producers showed a higher resistance rate to ampicillin, cefotaxime, ceftazidime, ciprofloxacin, cotrimoxazole, and amikacin. This higher rates of resistance of ESBLproducers to quinolones, cotrimoxazole, and aminoglycosides is in accordance with other studies $[15,22]$. The appearance of combined resistance to ampicillin and cotrimoxazole has become a common finding in ESBLproducing isolates in recent years as the genes responsible for resistance to these antibiotics are situated on the same plasmid [23]. None of the isolates in this study were found to be resistant to carbapenem. Mandal et al. [6] have also reported $100 \%$ sensitivity of ESBL-producing enterobacterial isolates from healthy populations towards imipenem and 4.85\% ESBL-producing E. coli resistant to piperacillintazobactam while $25.0 \%$ and $75 \%$ Klebsiella pneumoniae resistant to ciprofloxacin and cotrimoxazole, respectively. Baral et al. [24] documented resistance of $60 \%$ and $11 \%$ against ceftazidime and amikacin, respectively, in ESBLproducing $E$. coli isolates from school children.

Fecal commensal Enterobacterales carrying ESBLs are becoming increasingly commonly isolated from the fecal microbiota of healthy adults. In developing countries such as Nepal, antibiotics are easily accessible and many people use antibiotics without medical advice. This may encourage spreading of antibiotic-resistant enterobacterial commensals. The frequent presence of resistant commensal isolates in the gut may be a risk factor for prolonged infections and also enable propagation of resistance to other bacteria [25].

\section{Conclusions}

An alarmingly high prevalence of intestinal carriage of ESBL-producing enterobacterial commensal isolates was detected among HS students. There was a considerable number of such bacteria colonizing NHS students as well. The results confirm that healthy individuals may be an important reservoir for ESBL-producing bacteria, and this may pose a risk for the transmission of resistance throughout the wider community. Therefore, there is a need to plan and implement policies that may reduce their prevalence. Similar types of studies should be conducted among different medical and health sciences students in different parts of the country and different countries to establish the extent of carriage of ESBL-producing bacteria among such populations.

5.1. Limitations. This study represents data from students of only two educational institutions in Nepal. Although this was a cross-sectional study among different student groups, we were unable to access the risk factors associated with this high rate of fecal carriage of ESBL-producing isolates. Molecular analysis of ESBL-producers could not be performed.

\section{Abbreviations}

ASM:

AST:

American Society for Microbiology

Antibiotic susceptibility test

ATCC: $\quad$ American Type Culture Collection

BNS: $\quad$ Bachelor of Nursing Studies

B.Sc. MLT: Bachelor of Science in Medical Laboratory Technology

B.Sc. Nursing: Bachelor of Science in Nursing

CDT: $\quad$ Combined disk test

CLSI: Clinical and Laboratory Standards Institute 
ESBL: $\quad$ Extended-spectrum $\beta$-lactamase

HS: Health science

MHA: $\quad$ Mueller-Hinton agar

NHS: $\quad$ Non-health science

ZOI: Zone of inhibition.

\section{Data Availability}

Data will be made available if requested on justifiable reasons.

\section{Conflicts of Interest}

The authors declare that there are no conflicts of interest regarding the publication of this paper.

\section{Authors' Contributions}

BS and SKM contributed equally to this study. BS was responsible for study design, laboratory analysis, data entry and analysis, and preparation of the first draft of the manuscript. SKY was responsible for data analysis and interpretation and preparation of the second draft of the manuscript. SA was responsible for data interpretation and manuscript review. GRD was responsible for laboratory setup and manuscript review. SKM was responsible for study design, supervision, data analysis, and preparation of the final draft of the manuscript. All authors contributed toward drafting and revising the paper, gave final approval of the version to be published, and agreed to be accountable for all aspects of the work.

\section{Acknowledgments}

The authors acknowledge all the students who participated in this study, the principals of schools, management, and faculties of respective programs. The authors would also like to acknowledge Prof. Mark Willcox, Research Director of School of Optometry and Vision Science, UNSW Sydney, for editing the manuscript for English language.

\section{References}

[1] J. Carlet, "The gut is the epicentre of antibiotic resistance," Antimicrobial Resistance and Infection Control, vol. 1, pp. 1-7, 2012.

[2] A. Farra, T. Frank, L. Tondeur et al., "High rate of faecal carriage of extended-spectrum b-lactamase- producing Enterobacteriaceae in healthy children in Bangui, Central African Republic," Clinical Microbiology and Infection, vol. 22, pp. 891.e1-891.e4, 2016.

[3] A. Valverde, F. Grill, T. M. Coque et al., "High rate of intestinal colonization with extended-spectrum- $\beta$-lactamaseproducing organisms in household contacts of infected community patients," Journal of Clinical Microbiology, vol. 46, no. 8, pp. 2796-2799, 2008.

[4] A. Royden, E. Ormandy, G. Pinchbeck et al., "Prevalence of faecal carriage of producing Escherichia coli in veterinary hospital staff and students," Veterinary Record Open, vol. 6, pp. 1-14, 2019.
[5] A. Maharjan, A. Bhetwal, S. Shakya et al., "Ugly bugs in healthy guts! carriage of multidrug-resistant and ESBL-producing commensal Enterobacteriaceae in the intestine of healthy nepalese adults," Infection and Drug Resistance, vol. 11, pp. 547-554, 2018.

[6] D. K. Mandal, S. K. Sah, S. K. Mishra et al., "Carriage of extended-spectrum- $\beta$-lactamase- and AmpC- $\beta$-lactamaseproducing enterobacteriaceae (ESBL-PE) in healthy community and outpatient department (OPD) patients in Nepal," Canadian Journal of Infectious Diseases and Medical Microbiology, vol. 2020, Article ID 5154217, 9 pages, 2020.

[7] M. A. Islam, M. B. Amin, S. Roy et al., "Fecal colonization with multidrug-resistant $E$. coli among healthy infants in rural Bangladesh," Frontiers in Microbiology, vol. 10, pp. 1-9, 2019.

[8] S. H. Subramanya, I. Bairy, N. Nayak, S. Padukone, B. Sathian, and S. Gokhale, "Low rate of gut colonization by extendedspectrum $\beta$-lactamase producing Enterobacteriaceae in HIV infected persons as compared to healthy individuals in Nepal," PLoS One, vol. 14, no. 2, Article ID e0212042, 2019.

[9] H. Isenberg, Clinical Microbiology Procedure Handbook, American Society for Microbiology (ASM), Washington, DC, USA, 2nd edition, 2007.

[10] Performance Standards for Antimicrobial Susceptibility Testing, Twenty-Sixth Informational Supplement. CLSI Document M100S, Clinical and Laboratory Standards Institute, Wayne, PA, USA, 26th edition, 2016.

[11] P. M. A. Shanahan, C. J. Thomson, and S. G. B. Amyes, " $\beta$-Lactam resistance in normal faecal flora from South Africa," Epidemiology and Infection, vol. 115, no. 2, pp. 243-253, 1995.

[12] C. M. Lonchel, C. Meex, J. Gangoué-piéboji et al., "Proportion of extended-spectrum $\beta$-lactamase-producing Enterobacteriaceae in community setting in Ngaoundere, Cameroon," BMC Infectious Diseases, vol. 12, pp. 2-7, 2012.

[13] R. Cantón, A. Novais, A. Valverde et al., "Prevalence and spread of extended-spectrum $\beta$-lactamase-producing enterobacteriaceae in europe," Clinical Microbiology and Infection, vol. 14, pp. 144-153, 2008.

[14] O. O. Mahamat, A. Tidjani, M. Lounnas et al., "Fecal carriage of extended-spectrum $\beta$ - lactamase-producing enterobacteriaceae in hospital and community settings in Chad," Antimicrobial Resistance and Infection Control, vol. 8, pp. 1-7, 2019.

[15] U.-O. Luvsansharav, I. Hirai, M. Niki et al., "Prevalence of fecal carriage of extended-spectrum $\beta$-lactamase-producing Enterobacteriaceae among healthy adult people in Japan," Journal of Infection and Chemotherapy, vol. 17, no. 5, pp. 722-725, 2011.

[16] F. Ebrahimi, J. Mózes, J. Monostori et al., "Comparison of rates of fecal colonization with extended-spectrum beta-lactamase-producing enterobacteria among patients in different wards, outpatients and medical students," Microbiology and Immunology, vol. 60, no. 5, pp. 285-294, 2016.

[17] S. Islam, R. Selvarangan, N. Kanwar et al., "Intestinal carriage of third-generation cephalosporin-resistant and extendedspectrum $\beta$-lactamase-producing enterobacteriaceae in healthy US children," Journal of the Pediatric Infectious Diseases Society, vol. 7, no. 3, pp. 234-240, 2018.

[18] S. Karanika, T. Karantanos, M. Arvanitis, C. Grigoras, and E. Mylonakis, "Fecal colonization with extended-spectrum beta-lactamase-producing enterobacteriaceae and risk factors among healthy individuals: a systematic review and metaanalysis," Clinical Infectious Diseases, vol. 63, no. 3, pp. 310-318, 2016. 
[19] R. H. Bassyouni, S. N. Gaber, and A. A. Wegdan, "Fecal carriage of extended-spectrum $\beta$-lactamase- and AmpCproducing Escherichia coli among healthcare workers," The Journal of Infection in Developing Countries, vol. 9, no. 3, pp. 304-308, 2015.

[20] M.-H. Nicolas-Chanoine, C. Gruson, S. Bialek-davenet et al., "10-Fold increase (2006-11) in the rate of healthy subjects with extended-spectrum -lactamase-producing Escherichia coli faecal carriage in a Parisian check-up centre," Journal of Antimicrobial Chemotherapy, vol. 68, no. 3, pp. 562-568, 2013.

[21] R. Ben Sallem, K. Ben Slama, V. Estepa et al., "Prevalence and characterisation of extended-spectrum beta-lactamase (ESBL)-producing Escherichia coli isolates in healthy volunteers in Tunisia," European Journal of Clinical Microbiology \& Infectious Diseases, vol. 31, no. 7, pp. 1511-1516, 2012.

[22] D. Mathai, V. A. Kumar, B. Paul et al., "Fecal carriage rates of extended-spectrum $\beta$-lactamase-producing Escherichia coli among antibiotic naive healthy human volunteers," Microbial Drug Resistance, vol. 21, no. 1, pp. 59-64, 2015.

[23] S. Nys, I. N. Okeke, S. Kariuki, G. J. Dinant, C. Driessen, and E. E. Stobberingh, "Antibiotic resistance of faecal Escherichia coli from healthy volunteers from eight developing countries," Journal of Antimicrobial Chemotherapy, vol. 54, no. 5, pp. 952-955, 2004.

[24] B. P. Baral, B. Koirala, K. Gurung, R. R. Gurung, and H. Subramanava, "Fecal carriage of extended spectrum $\beta$ -lactamases (ESBL) producing Escherichia coli and Klebsiella spp. producing strains among school children in Pokhara, Nepal," Nepal Journal of Biotechnology, vol. 6, pp. 26-32, 2018.

[25] F. Rossi, F. Baquero, P. Hsueh et al., "In vitro susceptibilities of aerobic and facultatively anaerobic Gram-negative bacilli isolated from patients with intra-abdominal infections worldwide: 2004 results from SMART (Study for Monitoring Antimicrobial Resistance Trends)," Journal of Antimicrobial Chemotherapy, vol. 58, no. 1, pp. 205-210, 2006. 